\title{
Eliten unter sich
}

Wie Unternehmensberatungen ihre Macht über Städte entfalten

Unternehmensberatungen sind im Zuge der Neoliberalisierung über zwei Prozesse zu Akteuren der Politikgestaltung geworden: Einerseits haben sie selbst einen Markt in - ihrer Ansicht nach - ineffizient organisierten Behörden sowie wenig produktivitätsorientierten Regierungen entdeckt und ihre Angebote immer mehr für den öffentlichen Sektor ausgebaut. Andererseits hat die Verbreitung unternehmerischen Denkens, die Neoliberalisierung kennzeichnet, zur vermehrten Nachfrage nach ökonomisch strategischen Expert_innen geführt (Jupe/Funnell 2015, Resch 2005, Saint-Martin 2000). Ein dadurch steigender Einfluss von ,management consultants" ist auch auf Ebene der Stadtpolitik sichtbar geworden. Sie sind inzwischen Teil eines elitären Geflechts, das Stadtentwicklung maßgeblich mitbestimmt.

Die Frage wer macht eigentlich eine Stadt? führt allerdings notwendigerweise in ein nahezu unüberschaubares Dickicht von verschiedenen Akteuren - von institutionellen Organisationen über informelle Initiativen bis hin zu jeder einzelnen Person, die in der Stadt lebt. Wenn nun von elitären Machtverhältnissen gesprochen wird, bedeutet es deshalb nicht, dass alle anderen Formen der Stadtproduktion ausgehebelt sind. Im Gegenteil: gerade in Zeiten, in denen die Legitimität bestehender Verhältnisse zum Beispiel durch soziale Bewegungen oder ökonomische Krisen in Frage gestellt wird, geraten auch bestehende elitäre Netzwerke ins Wanken. Allerdings setzen diese auch gerade in diesen Momenten spezifische Machttechniken ein, die die wackelnde Macht wieder stabilisieren sollen.

Im Folgenden möchte ich vier solcher Machttechniken vorstellen, die in einer empirischen Studie über die Bedeutung von Unternehmensberatungen in der strategischen Stadtentwicklung sichtbar geworden sind (ausführlich dazu Vogelpohl 2018a; b, Vogelpohl/Klemp 2018). Untersucht habe ich sechs deutsche Städte beziehungsweise Regionen, die zwischen 1999 und 2014 von den Unternehmungsberatungen McKinsey oder Roland Berger in der Suche nach Auswegen aus einer tatsächlichen oder wahrgenommenen ökonomischen Krise unterstützt wurden. In der Regel war ein ökonomischer Umbruch (die Abwanderung eines großen Unternehmens oder weiterreichende Veränderungen des Arbeitsmarktes durch Deindustrialisierung) 
die kritische Ausgangslage, aus der die consultants heraushelfen sollten oder wollten. Denn nicht alle Beratungsprojekte sind von den städtischen Regierungen ausgegangen und an McKinsey oder Roland Berger vergeben worden. In den von mir untersuchten Fällen traf dies nur in Dortmund, Halle (Saale), Essen und Goslar/Osterode zu. In Hamburg und Berlin hingegen hat jeweils McKinsey ungefragt und unbezahlt ein pro bono-Projekt zur Stadtentwicklung gestartet. Die fehlende monetäre Gegenleistung macht die Frage nach der Machtstrategie hinter städtischen Beratungsprojekten besonders drängend.

\section{Neue Netzwerke: „Das Netzwerk, was man nach so einem Projekt hat, ist natürlich immens“}

Um Informationen über aktuelle Entwicklungen, aber auch über Ideen für zukünftige Politikideen zu erhalten, stehen zu Beginn aller Beratungsprojekte für stadtstrategische Fragen umfangreiche Analysen. Für eine qualitative Analyse sprechen die Berater_innen mit vielen „Meinungsbildnern“ (McKinsey Hamburg 2001: 2). Um die hundert Gespräche pro Stadt wird oft als Zahl genannt. Die ,Meinungsbildner sind beispielsweise Geschäftsführer_innen oder Führungskräfte in wirtschaftlichen Unternehmen, der Politik, den Medien, gegebenenfalls der Kirche oder lokal wichtigen Vereinen. Oft werden darüber hinaus noch Diskussionsveranstaltungen unter den Interviewten organisiert, auf denen, die Zukunft der Stadt' erörtert wird. So entstehen mithilfe der Berater_innen neue elitäre Netzwerke, von denen eine dynamischere, zielorientierte Stadtentwicklung ausgehen soll.

Elitär ist das neu gebildete Netzwerk aus zwei Gründen, die im Selbstverständnis der Berater_innen wurzeln. Erstens, so drückt es ein Mitarbeiter der Dortmunder Stadtverwaltung aus, ,arbeiten die McKinseys immer top down“(DO11_verw[1]). Dahinter steckt die Annahme, dass Ziele sich vor allem dann verwirklichen lassen, wenn die letztendlichen Entscheidungsträger_innen davon überzeugt sind. In den Landkreisen Goslar und Osterode wurde das in der Verwaltung so wahrgenommen:

„Die sprechen nicht mit [denen] da unten, sondern die fangen immer oben an den Köpfen an und sei es nur eine Vorbereitung einer Aufsichtsratssitzung oder von Lenkungsausschusssitzungen ... Die fangen natürlich, auch Kraft ihrer Beratungsmacht, sage ich jetzt einfach mal, immer von oben an. Die bequatschen dann die Führung und die sagen dann: ,Ja, das ist toll.““(GOS36a_verw)

Zweitens integrieren die Berater_innen nicht nur die regierenden Parteien und nicht nur die oberste Riege der administrativen Leitungseinheiten, sondern auch die in der zweiten oder dritten Reihe. Auf diese Weise schaffen sie ein relativ langfristiges Netzwerk aus Personen in machtvollen Positionen, das auch nach Wahlen oder nach Führungswechseln bestehen bleibt und deswegen lang über das eigentliche Beratungsprojekt hinaus Bestand hat.

Zugleich erschließt sich das Beratungsunternehmen selbst mit den Interviews eine Vielzahl an Kontakten. Das in der Überschrift genannte Zitat eines Beraters, der für das Unternehmen Roland Berger in Halle (Saale) aktiv war, nimmt darauf Bezug: „Das Netzwerk, was man nach so einem Projekt 
hat, ist natürlich immens. Man spricht mit allen großen Unternehmen, den Geschäftsführern, der Gesellschaft ... Und so lernt man die alle kennen“ (HAL24_Berat). Da die Beratungsunternehmen (wie auch viele der lokal angesiedelten einflussreichen Akteure) global agieren, hat die Machttechnik, neue Netzwerke zu spinnen, oft eine nationale, manchmal sogar internationale Reichweite.

\section{Neues Vokabular: „Informationen aufgepeppt“}

Neben der Kritik an der Konzentration von Entscheidungsmacht ist eine häufige Kritik an externer Beratung, dass inhaltlich kaum neue Probleme oder Ideen für Lösungen benannt werden. Auch wenn allein die Bündelung von Informationen schon eine Veränderung einer Problemwahrnehmung nach sich ziehen kann, ist einezentrale Veränderung durch Beratung die Einführung eines neuen Vokabulars. Während die Idee von Wirtschaftswachstum, Wettbewerb oder Clustern an sich nicht neu und vielen städtischen Politiker_innen ohnehin bekannt ist, erzeugen neue Bezeichnungen einen anderen Druck, in diesem Wettbewerb aktiver zu agieren.

Die Beratungsstudie für Berlin (McKinsey Berlin 2010) ist beispielsweise durchzogen von Begriffen wie ,Führungsposition“ oder ,Modellstadt in Wissenschaft und Industrie. Mit diesem Vokabular wird ein starker Wettbewerbsdruck erzeugt, sich vor allem international zu vergleichen und an internationalen Rankings und Zahlen zu orientieren. In einem anderen Beispiel, in Goslar und Osterode, wird nicht mehr technisch von Ressourcenoder Energietechnologie, sondern von „Natur trifft Technik“ gesprochen (Initiative Zukunft Harz 2011). Dies soll eine neue Identifikation und Begeisterung für bestehende Entwicklungen entfachen in einer Region, die eigentlich von Schrumpfung betroffen ist (vgl. Bernt/Weck 2012). Und die Überschriften der Projektergebnisse verweisen regelmäßig auf eine (andere) Zukunft: „Hamburg Vision 2020‘, ,Berlin 2020“ oder,Essen.2030` werden die Projekte betitelt. So wird auf Veränderung gepocht und ein Handlungszwang erzeugt.

Verstärkt wird diese Machttechnik der Einführung eines neuen Vokabulars, die ein Politiker aus Osterode im obigen Eingangszitat ,Aufpeppen“von Informationen nennt (GOS35_polit), durch eine eindrückliche Wort- und Bildsprache (vgl. Vogelpohl 2015). Dies ist letztlich auch an die Medien adressiert. Diese greifen Diagnosen, dass das Problem „weniger im Produkt [Halle (Saale)] als im Image" (Roland Berger 2002, 21) liege, oder spektakuläre Versprechen wie „500.000 neue Jobs“ in nur 10 Jahren (McKinsey Berlin 2010, 15) gerne und schnell aufgreifen. Die von den Beratungen formulierten, klar und einfach erscheinenden Ziele erzeugen so einen politischen Druck, sich den dort thematisierten Problemen mit finanziellen und personellen Mitteln zu widmen (und dabei andere Felder, die außerhalb der Problemwahrnehmung der Unternehmensberater_innen liegen, zu vernachlässigen). 


\section{Neue Methoden: „Sie bringen Know-how rein, Methodenkompetenz"}

Die „Methodenkompetenz“, die in oben genanntem Zitat von einem Beteiligten aus Dortmund angesprochen wird (DO11_verw), prägt zentral und langfristig die Art und Weise, wie Stadtentwicklung gedacht und organisiert wird. Sie wird in fast jedem Interview in allen Fallstudien angesprochen. Dahinter verbirgt sich zunächst die Dimension einer relativ klassischen, aber breit angelegten Stärken-Schwächen-Analyse. Die in einen internationalen Vergleich gestellten Zahlen zum Beispiel zu lokaler Bruttowertschöpfung oder Start-Up-Quoten dienen dann mittel- und langfristig als Parameter für Stadtentwicklungsprojekte. Ergänzt wird dieses „number crunching“ (HH32_berat) durch Methoden des Projektmanagements, das heißt durch klar abgegrenzte und definierte Probleme, Indikatoren, Ziele und Maßnahmen.

Die Konsequenz aus einer solch systematischen Herangehensweise an Gegenwart und Zukunft der Stadt ist eine ,Projektisierung des Städtischen“ (vgl. Vogelpohl 2018b). Das ,Projekt Stadt‘ erscheint somit effektiv und relativ einfach zu managen zu sein: Es gibt eine eindeutige Problemanalyse, es werden vier bis fünf Kernziele als Lösungen formuliert für die wiederum jeweils vier bis fünf Einzelprojekte benannt werden. Das Management-Denken aus Unternehmen wird von den Berater_innen also relativ linear auf Städte übertragen. Der wichtigste Berater für das von McKinsey organisierte dortmund project hat dies auch in einem Buch über „Strategisches Management von Städten“ (Weig 2004) zusammengefasst. Die Projektisierung des Städtischen wird langfristig angelegt und abgesichert, indem oftmals Mitarbeiter_innen in den lokalen Behörden während des Beratungsprojektes in diesem analytisch-strategischen Denken geschult werden.

Die Etablierung neuer Methoden in der strategischen Stadtentwicklung ist eine elitäre Machttechnik, weil systematisch die thematische und teilweise konfliktbehaftete städtische Komplexität ausgeblendet wird. Durch eine methodisch klar angeleitete Sortierung von Themen werden scheinbar alle Widersprüche ausgeräumt. Diese inhaltliche Reduktion des Städtischen ist begleitet von einer beschleunigten Projektentwicklung, in der schlicht auch nicht die Zeit für kontroverse Debatten bleibt. Sinnvolle Partizipation - zumal eine, die auch Konflikte ernst zu nehmen anstatt zu beschwichtigen weiß (Klöti 2016) - kann in einer projektisierten Stadt ebenso wenig Platz finden wie mittel- oder langfristige Ziele eines sozialen Miteinanders.

\section{Neue Aktivität: „Regionales Durchschütteln“}

Die neu gebildeten Netzwerke können auch ohne politische Mitwirkung aktiv werden. Mit neuen Clustern, Förderprogrammen, Schwerpunktveranstaltungen oder sogar neu gebildeten Institutionen ist dies jedoch noch effektiver. Die Beratungsprojekte zielen deshalb in jedem Fall auf eine lokale und regionale Furore rund um ökonomische Potenziale. „Regionales Durchschütteln“ (GOS36b_verw) nennt das ein Mitarbeiter aus der Stabstelle der neuen Organisationseinheit „Initiative Zukunft Harz“ in Goslar und Osterode. Der Aufruf für eine „proaktive Einstellung“ (McKinsey Berlin 2010: 50) sowie 
für ein koordiniertes Engagement steht oftmals als Gesamtfazit am Ende der Beratungsdokumente. Dies zeigt, dass eine neue Aktivität aus Sicht der Beratenden letztlich entscheidend ist - entscheidender als konkrete Ideen für anzugehende Projekte oder neu zu bildende Cluster.

Eine neue Dynamik, die sich aus politökonomischen Netzwerken und planerisch-strategischen Maßnahmen speist, ist nicht zuletzt für die Unternehmensberatungen selbst von zentralem Interesse. Denn wenn spezifische Branchen gefördert werden und ein besonderes Wachstum verzeichnen, vergrößern consultants ihren eigenen Markt. Demzufolge stehen auch nicht unbedingt die geeignetsten Branchen in den Studien der Unternehmensberatung im Fokus, sondern jene, in denen sie sich auskennen und die ihre Kundschaft bilden. In Berlin beispielsweise war die Idee des leitenden Beraters, die Studie unter anderem auf nachhaltige Mobilität zu fokussieren, innerhalb von McKinsey nicht durchsetzbar. Stattdessen wollte das Berliner Büro E-Mobilität mit Schwerpunkt auf den Individualverkehr im Fokus sehen - eine Branche, für die McKinsey auch sonst beratend aktiv ist.

Die Begriffe ,Durchschütteln' und ,neue Aktivität' sind auch mit einer Idee von Auf- oder Wachrütteln konnotiert. Die genannten Beispiele zeigen allerdings, dass das aufgerüttelte Themenfeld sehr selektiv ist und von nur wenigen Akteuren bespielt wird. Ökonomisches Wachstum ist fast immer zentral, während zum Beispiel Fragen des Wohnens, von ungleichen Zugangschancen oder Ökologie in den von mir untersuchten Beispielen kaum angesprochen werden. Diese Selektivität ist umso schwerwiegender, weil sie durch die Aufmerksamkeit des Beratungsprojektes gestärkt und so noch wirkmächtiger wird. Eine neue politische und mediale Aktivität ist also der Weg, auf dem sich die neuen Netzwerke schließlich materialisieren können. Ein Machtfeld, das durchgeschüttelt und in Bewegung geraten ist, ist die Grundlage für neue sowie sich intensivierende Koalitionen.

\section{Fazit: Politikberatung als Machtinstrument}

Gerade die großen Unternehmensberatungen sind bekannt für ihre enorm hohen Stunden- und Tagessätze (Leif 2006). Die von mir untersuchten Beratungen für Städte und Regionen sind allerdings entweder pro bono gewesen, also unbezahlte und unbeauftragte Projekte, oder haben nur relativ wenig gekostet verglichen mit Projekten in transnationalen Unternehmen. Es stellt sich daher die Frage: Wieso werden die Firmen politikberatend tätig, wenn es sich finanziell wenig lohnt?

Die Antwort liegt fast ausschließlich im Ausbau politischer und ökonomischer Macht: Erstens kann die Unternehmensberatung über das Beratungsprojekt Kontakte zu Unternehmen in der Stadt bzw. der Region herstellen. Gleichzeitig werden während des Projektes Beziehungen zwischen Unternehmen ausgebaut. So erstarkt die ökonomische Basis für weitere, dann in der Regel gut bezahlte private Beratungsprojekte. Zweitens wird gezielt die Aufmerksamkeit der Medien gesucht. Auf diese Weise werden lokalpolitische Diskurse ganz im Sinne der Wachstumsideen des Beratungsunternehmens geprägt. Drittens werden auch Persönlichkeiten aus der Zivilgesellschaft befragt, die schließlich die Ergebnisse der Beratung als Befürworter_innen und Multiplikator_innen in die Stadtgesellschaft tragen sollen. Viertens 
wird politisches Wohlwollen für die zentralen Ideen des Projektes generiert. Denn politisch mächtige Personen übernehmen teilweise selbst zentrale Funktionen im Beratungsprozess, so dass sie das Projekt entsprechend als ,ihr' Projekt begreifen und nachhaltig vorantreiben. Und nebenbei werden noch junge Mitarbeiter_innen der Beratungsunternehmen in relativ wenig entscheidenden Fällen geschult oder zeitliche Lücken zwischen größeren Projekten überbrückt.

Ein stadtpolitisches Beratungsprojekt ist also in erster Linie ein Vehikel politökonomischer Eliten für die Entfaltung und Stabilisierung ihrer Machtposition. Dass dieses Vehikel jedoch reibungslos städtische Zukünfte strukturiert, ist damit noch nicht gesagt. Denn Koalitionen bilden auch Kritiker_innen, sei es die politische Opposition oder seien es lokale Aktivist_innen. Und auch alternative Diskurse zum Beispiel über eine soziale oder umweltgerechte Stadt werden nicht ausgehebelt und können als Antwort auf die wachstumsorientierte Vision der Beratungsunternehmen möglicherweise sogar neuen Schwung erhalten. Allerdings sind die sorgfältig vorbereiteten Machttechniken der management consultants effektiv und schwer ausweichbar. Sie fordern Antworten heraus. Diese fallen in einem dafür positiv gestimmten Umfeld zumeist ganz im Sinne der Unternehmensberatung und ihren politischen und ökonomischen Partner_innen aus.

\section{Endnoten}

[1] Die Interviewkürzel verweisen auf 1. das Fallbeispiel (DO: Dortmund; GOS: Goslar/ Osterode, HAL: Halle; HH: Hamburg), 2. das spezifische Interview (durchnummertiert) sowie 3. den Funktionsbereich der_s Interviewten (verw: Verwaltung; berat: Beratung; polit: Politik).

\section{Autor_innen}

Anne Vogelpohl ist Geographin und beschäftigt sich mit Stadtpolitik in Hinblick auf Beratung, Wohnen sowie Arbeit und nutzt feministische Methodologien.

anne.vogelpohl@haw-hamburg.de

\section{Literatur}

Bernt, Matthias / Weck, Sabine (2012): Peripherisierung, Schrumpfung und Governance: Handlungsansätze der Stadtpolitik in sechs deutschen Mittelstädten. In: Michael Haus / Sabine Kuhlmann (Hg.), Lokale Politik und Verwaltung im Zeichen der Krise? Wiesbaden: VS, 256-273.

Initiative Zukunft Harz (Hg.) (2011): Natur trifft Technik. Überblick über die Handlungsfelder und Projektbündel. Clausthal-Zellerfeld.

Jupe, Robert / Funnell, Warwick (2015): Neoliberalism, consultants and the privatisation of public policy formulation: The case of britain's rail industry. In: Critical Perspectives on Accounting 29, 65-85.

Klöti, Tanja (2016): Zum Verhältnis von partizipativer Stadtentwicklung, neoliberaler Stadtpolitik und stadtteilbezogener Sozialer Arbeit. In: Patrick Oehler / Nicola Thomas / Matthias Drilling (Hg.), Soziale Arbeit in der unternehmerischen Stadt: Kontexte, Programmatiken, Ausblicke. Wiesbaden: Springer VS, 53-73. 
Leif, Thomas (2006): Beraten \& verkauft: McKinsey \& Co. - der große Bluff der Unternehmensberater. München: C. Bertelsmann.

McKinsey Berlin (2010): Berlin 2020 - Unsere Stadt. Wirtschaftliche Perspektiven durch neue Wachstumskerne. Berlin: McKinsey \& Company.

McKinsey Hamburg (2001): Hamburg Vision 2020 - Vom nationalen Zentrum zur Europäischen Metropole. Hamburg: McKinsey \& Company.

Resch, Christine (2005): Berater-Kapitalismus oder Wissensgesellschaft? Zur Kritik der neoliberalen Produktionsweise. Münster: Westfälisches Dampfboot.

Roland Berger (2002): Abschlußbericht Investorenansiedlungs- und Stadtmarketingkonzept. Halle: Roland Berger.

Saint-Martin, Denis (2000): Building the New Managerialist State - Consultants and the Politics of Public Sector Reform in Comparative Perspective. Oxford/New York: Oxford University Press.

Vogelpohl, Anne (2015): RaumBilder und Wirtschaft - Visuelle Strategien in der Wirtschaftsförderung. In: Judith Miggelbrink / Antje Schlottmann (Hg.), Visuelle Geographien - Zur Produktion, Aneignung und Vermittlung von RaumBildern. Bielefeld: transcript, 121-132.

Vogelpohl, Anne (2018a): Consulting as a threat to local democracy? Flexible management consultants, pacified citizens, and political tactics of strategic development in german cities. In: Urban Geography, 39, 1345-1365.

Vogelpohl, Anne (2018b): Global expertise, local convincing power: Management consultants and preserving the entrepreneurial city. In: Urban Studies, doi: 10.1177/0042098018768490.

Vogelpohl, Anne / Klemp, Felicitas (2018): The creeping influence of consultants on cities: McKinsey's involvement in Berlin's urban economic and social policies. In: Geoforum 91, 39-46.

Weig, Florian (2004): Balanced Scorecard für Strategisches Management von Städten - Ein Ansatz unter Einsatz von Internetumfragen. Wiesbaden: VS. 
